\title{
Using STEM to Probe the in-situ Dynamics of Multimetallic Nanoparticles Grown in Polymer Nanoreactors
}

Jingshan S. Du ${ }^{1,3}$, Peng-Cheng Chen ${ }^{1,3}$, Vinayak P. Dravid ${ }^{1,3,4}$ and Chad A. Mirkin ${ }^{1,2,3}$

1. Department of Materials Science and Engineering, ${ }^{2 .}$ Department of Chemistry, and ${ }^{3 .}$ International Institute for Nanotechnology, Northwestern University, Evanston, IL 60208, United States

4. NUANCE Center, Northwestern University, Evanston, IL 60208, United States

Although there has been much progress, the dynamics of nanoparticle (NP) growth in liquids and the nature of liquid-particle interactions during the growth process are poorly understood. This is especially true when studying the evolution of multimetallic NPs in liquid environments or polymer matrices [1]. The growth trajectory of NPs and several inter-particle dynamic phenomena, such as Ostwald ripening, migration-coalescence, and oriented attachment, have been observed using liquid-cell in-situ (scanning) transmission electron microscopy $[(\mathrm{S}) \mathrm{TEM}]$ techniques [2,3]. Evaporation or decomposition of a dispersant thin film on a surface can drive NPs either to self-assemble into 2D monolayers and complicated nanopatterns, or to coarsen and form islands and thin solid films. This evaporation-driven material deposition is often spatially nonuniform at the nanometer scale [4]. The formation process and kinetics of such nonuniformity at the nanoscale have been difficult to directly monitor, since imaging such interactions by in-situ electron microscopy often suffers from the low contrast and fast evaporation of most liquids. Polymer nanoreactors made by Dip-Pen Nanolithography (DPN) have been proven to be a powerful emerging approach to control the formation of multimetallic NPs by thermally driven growth and coarsening [5]. Due to the high decomposition temperature, polymer nanoreactors have also provided a relatively stable fluidic environment to study NP dynamics using STEM [6] (Figure 1A). Herein, we report a study focused on the growth of Au-Pt NPs and their coarsening kinetics driven by polymer decomposition. The process is followed by in-situ STEM on thin sections of polymer nanoreactors, which are patterned on electron-transparent amorphous carbon films.

Hemispherical domes of the polymer, poly(ethylene oxide)-block-poly(2-vinylpyridine) (PEO- $b$-P2VP) coordinated with metal precursors, are deposited on amorphous carbon films using DPN (Figure 1B). Atomic force microscopy (AFM) show that the as-patterned domes have high aspect ratios (ca. 9.0) on carbon films with contact angles around $13^{\circ}$, resulting in ultra-thin sections (less than $20 \mathrm{~nm}$ near the edge) (Figure 1C and D). These polymer nanoreactors are cooled to liquid nitrogen temperature in a Hitachi HD-2300 STEM operated at $200 \mathrm{kV}$. Under electron radiation, the polymer exhibits fluidic characteristics. At relatively low electron dose rates, the growth of Au-Pt NPs is observed with a bimodal distribution of particles, differentiated by diameter (Figure 2A). Experiments performed at higher electron dose rates reveal that polymer decomposition is not uniform at the nanoscale. Similar to water drying on a hydrophilic surface on the macroscale, formation of nanoscale voids is observed leading to thin polymer filaments. These filaments decompose rapidly according to high-angle annular dark field (HAADF) signal quantification studies (stage II, Figure 2B). Such voiding behavior drives the NPs to migrate and coalesce with each other (Figure 2C). When the polymer is sufficiently thin between adjacent NPs (at ca. 1-nm scale), the NPs are observed to conjugate with a sudden acceleration. This observation is consistent with the conclusion that asymmetric capillary forces at the nanoscale drive the NPs to coalesce and form larger particles, which are dispersed nonuniformly on the surface. 
This presentation will introduce recent advances in the formation of multi-metallic NPs in polymer nanoreactors derived from DPN and related nanopatterning approaches. This will be followed by a description of mechanistic studies of their dynamics using in-situ electron microscopy. The work underscores how these novel polymer nanoreactors can be used to synthesize multimetallic nanoparticles with extraordinary precision and as a tool, when combined with in-situ STEM, for understanding how such structures form, at least under the conditions of an electron beam [7].

\section{References:}

[1] G V Ramesh, et al, Chemical Society

Reviews 38 (2009), p. 2646.

[2] H-G Liao, et al, Science 345 (2014), p. 916.

[3] D Li, et al, Science 336 (2012), p. 1014.

[4] E Rabani et al, Nature 426 (2003), p. 271.

[5] P-C Chen et al, Science 352 (2016), p. 1565.
[6] J Chai et al, Journal of the American

Chemical Society 134 (2012), p. 158.

[7] The authors acknowledge funding from GlaxoSmithKline (100037477) and the Air Force Research Laboratory (FA8650-15-25518).
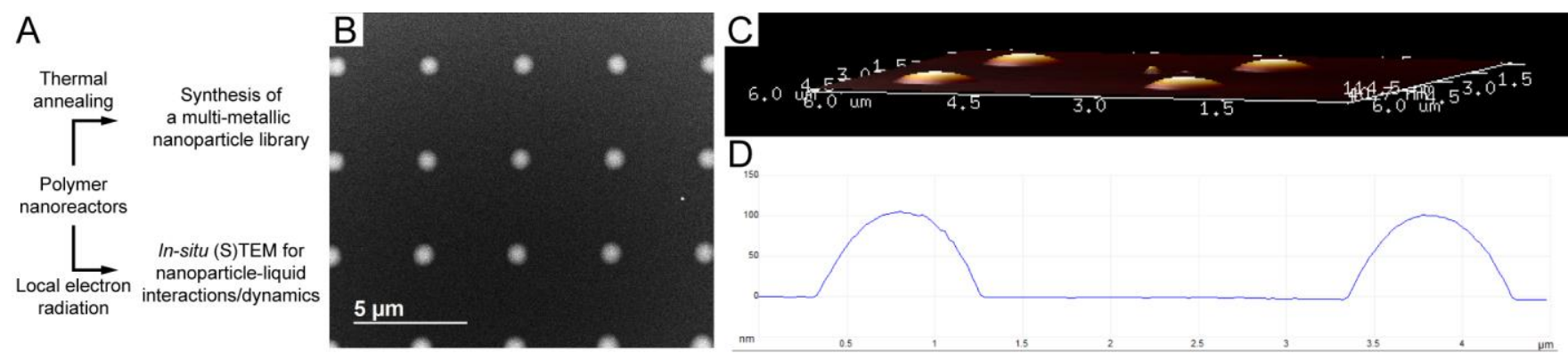

Figure 1. (A) Schematic chart showing polymer nanoreactors for both synthesis and the in-situ study of nanoparticles. (B) HAADF image showing a nanoreactor array on a carbon film. (C) AFM 3D morphology and (D) height profile of nanoreactors on a carbon film.

A

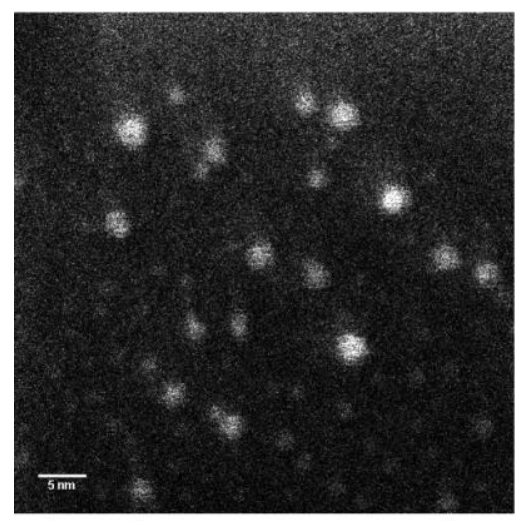

B

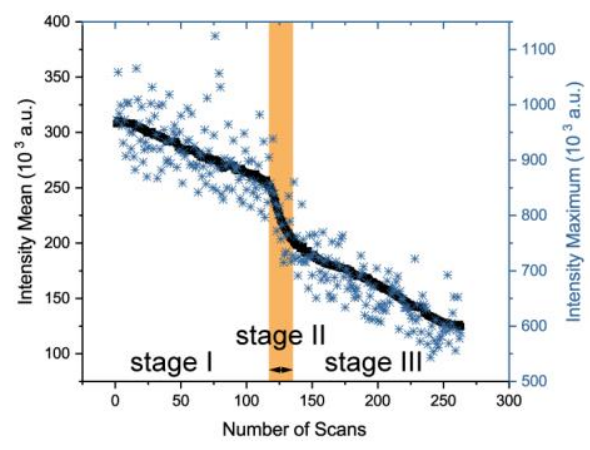

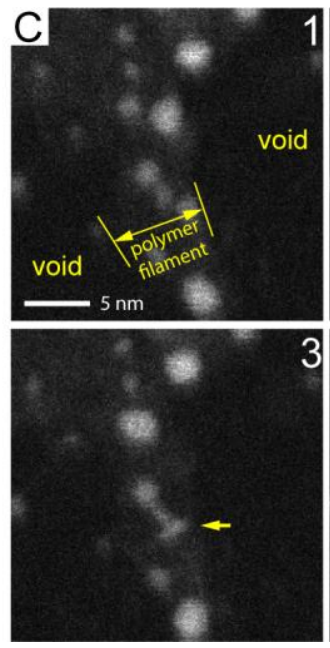

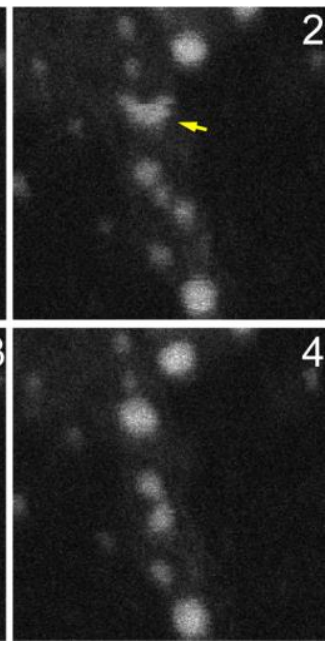

Figure 2. Electron radiation-induced NP formation, polymer decomposition and the beam-induced coarsening of NPs in polymer nanoreactors. (A) Au-Pt NPs grown under a low electron dose rate. (B) HAADF intensity quantification of decomposing PEO- $b$-P2VP under electron probe scanning. (C) Sequential HAADF images showing migration and coalescence of Au-Pt NPs (arrows showing accelerated coalescences) driven by polymer decomposition. All images have a scale bar of $5 \mathrm{~nm}$. 